\title{
EFFECT OF HELICITY ON THE BUCKLING BEHAVIOR OF SINGLE-WALL CARBON NANOTUBES
}

\author{
JEEHYANG HUH ${ }^{1}$, HOON HUH ${ }^{2 \dagger}$ \\ KAIST, Daejeon, KOREA, \\ utopias62@kaist.ac.kr,hhuh@kaist.ac.kr
}

Received 15 June 2008

Revised 23 June 2008

\begin{abstract}
Simulations of single-wall carbon nanotube(SWCNT)s having a different chiral vector under axial compression were carried out based on molecular dynamics to investigate the effect of the helicity on the buckling behavior. Calculation was performed at room temperature for $(8,8)$ armchair, $(14,0)$ zigzag and $(6,10)$ chiral single-wall carbon nanotubes. The Tersoff potential was used as the interatomic potential since it describes the $\mathrm{C}-\mathrm{C}$ bonds in carbon nanotubes reliably. A conjugate gradient (CG) method was used to obtain the equilibrium configuration. Compressive force was applied at the top of a nanotube by moving the top-most atoms downward with the constant velocity of $10 \mathrm{~m} / \mathrm{s}$. The buckling load, the critical strain, and the Young's modulus were calculated from the result of MD simulation. A zigzag carbon nanotube has the largest Young's modulus and buckling load, while a chiral carbon nonotube has the lowest values.
\end{abstract}

Keywords: Molecular dynamics; single-wall carbon nanotube; buckling behavior; helicity.

\section{Introduction}

After discovered first by S. Iijima ${ }^{1}$ in 1991 , carbon nanotubes (CNTs) are widely used in Micro-Electro-Mechanical Systems (MEMS) and Nano-Electro-Mechanical Systems $(\mathrm{NEMS})^{2}$ since they have good mechanical, ${ }^{3,4}$ electrical, ${ }^{5}$ and magnetic ${ }^{6}$ properties.

It is essential to obtain the mechanical properties of CNTs, such as the Young's modulus and the buckling load, for its application to mechanical systems. Many researchers have obtained the mechanical properties of CNTs through experimental measurements or computer simulations. Quantum or molecular level simulation has been of great interest to obtain the properties with computer simulations, since continuum level simulation does not reproduce the atomistic behavior correctly. Yakobson et al. ${ }^{4}$ observed the mechanical behavior of single-wall carbon nanotubes (SWCNTs) under large deformation by molecular dynamics (MD). Ozaki et al. ${ }^{7}$ used a tight binding (TB) method to investigate the behavior of SWCNTs under axial load. Buehler et al. ${ }^{8}$ studied buckling behavior of SWCNTs with various aspect ratios and Dereli et al. ${ }^{9}$ investigated a $(10,10)$ SWCNT under compressive loading with a TB method. Most of the researchers

Corresponding Author. 
focused mainly on the behavior of armchair CNTs and few studies investigated the effect of the helicity. A study on the effect of the helicity can be meaningful since CNTs have different electric properties according to their helicity. ${ }^{10}$ Ozaki et al. ${ }^{7}$ reported that elastic moduli are insensitive to the size and the helicity. However, $\mathrm{Lu}^{11}$ found that the $0 \mathrm{~K}$ stress of SWCNTs is remarkably sensitive to the helicity. Since those two results are contrary to each other, further investigation on the effect of the helicity on the mechanical behavior of CNTs is required.

This study observes the mechanical behavior of SWCNTs having different helicity under axial compression through molecular dynamics simulations with the Tersoff potential function. ${ }^{12}$ The Young's modulus, the critical strain, and the buckling load are calculated for each tube comparing with other literatures.

\section{Theoretical Backgrounds}

\subsection{Carbon nanotubes}

A carbon nanotube (CNT) is a wrapped graphene sheet that has a diameter of a few nanometers. There exist CNTs with various dimensions. A CNT can be classified into two groups: single-wall nanotubes (SWCNT) and multi-wall nanotubes (MWCNT). SWCNTs consist of one graphite sheet, whereas MWCNTs consist of more than one SWCNT piled up into a single tube.

A SWCNT can be classified into armchair, zigzag and chiral nanotubes according to their helicity, or the chiral vector. ${ }^{10}$ The chiral vector $\mathbf{C}_{\mathbf{h}}$ is a pair of indices $(n, m)$ which represents the way the graphene sheet is wrapped as in Fig. 1

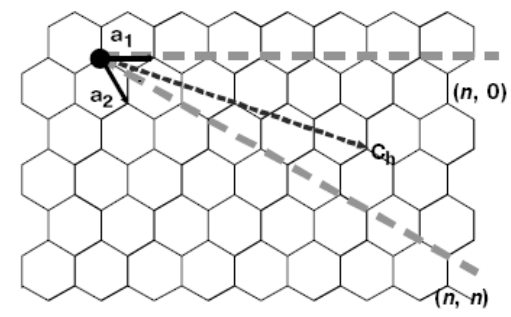

Fig. $1 .{ }^{10}$ Schematic of a two-dimensional graphene sheet illustrating lattice vectors $\mathbf{a}_{1}$ and $\mathbf{a}_{2}$, and the roll-up vector $\mathbf{c}_{\mathbf{h}}=n \mathbf{a}_{1}+m \mathbf{a}_{2}$.

The integers $n$ and $m$ denote the number of unit vectors along two directions in the honeycomb crystal lattice of graphene. Nanotubes are called "zigzag" if $m=0$, "armchair" if $n=m$, and "chiral" otherwise.

\subsection{Molecular dynamics}

Molecular dynamics (MD) is a type of computer simulation to predict the motion of atoms and molecules by time integration of the known equations of motion. Conventional MD uses the Hamiltonian equation (Eq. (1) $)^{13}$ as the equations of motion. 


$$
\mathbf{p}_{i}=-\frac{\partial H}{\partial \mathbf{q}_{i}}, \mathbf{q}_{i}=-\frac{\partial H}{\partial \mathbf{p}_{i}}
$$

where $H$ is Hamiltonian of the system, $\mathbf{q}_{\mathrm{i}}$ and $\mathbf{p}_{\mathrm{i}}$ are the generalized position vector and the generalized momentum vector respectively. Hamiltonian contains the kinetic energy and the potential energy.

A different material has different potential, so it is very important to select an appropriate potential function ${ }^{14}$ that is appropriate for each material. Among various potentials, density dependent potential functions ${ }^{15}$ or multi-body potential functions ${ }^{12,16}$ are generally used for metals and ceramics. In this paper, the Tersoff potential function ${ }^{12}$, a type of multi-body potential functions, was used.

\section{Simulation Methods}

Buckling simulation of SWCNTs under axial compression was carried out based on molecular dynamics simulation to investigate how the buckling behavior of nanotubes changes according to the helicity. MD simulation was performed for $(8,8)$ armchair, $(14,0)$ zigzag, $(6,10)$ chiral SWCNTs. Chiral vectors are carefully selected that those three nanotubes have similar dimensions, that is, a diameter of around $11 \AA$ and a length of around $80 \AA$. Positions of atoms were generated by setting the initial C-C bond length to be $1.42 \AA$. Initial dimensions of each nanotube are shown in Table 1 .

Table 1. Initial dimensions of the nanotubes.

\begin{tabular}{c|ccc}
\hline Chiral vector & $D(\AA)$ & $L(\AA)$ & Aspect ratio $($ L/D $)$ \\
\hline$(8,8)$ & 10.8 & 79.934 & 7.4 \\
$(14,0)$ & 10.94 & 76.325 & 6.977 \\
$(6,10)$ & 10.96 & 78.8087 & 7.19 \\
\hline
\end{tabular}

The interaction among atoms was described by the Tersoff potential function, ${ }^{12}$ which is known to describe the C-C bond in CNTs reliably. ${ }^{8}$ The Tersoff potential is described as Eq. (2):

$$
V_{i j}=f_{C}\left(r_{i j}\right)\left[a_{i j} f_{R}\left(r_{i j}\right)+b_{i j} f_{A}\left(r_{i j}\right)\right.
$$

where $f_{R}$ is the repulsive potential, $f_{A}$ is the attractive potential, $f_{C}$ is a smooth cut-off function, and $a_{i j}, b_{i j}$ are the coefficient to consider the effect of the bond order.

LAMMPS $^{\dagger}$, a classical molecular dynamics code, was used to carry out the MD simulation and VMD $\$$, a visualization program, was used for visualization. The time step was set to be $1 \mathrm{fs}$, and the simulation was carried out at room temperature. Firstly, energy minimization was carried out with the conjugate gradient (CG) method. Secondly, the atoms at the top were moved downwards with the constant velocity of $10 \mathrm{~m} / \mathrm{s}$ to apply axial compression while the atoms at the bottom were fixed. Force-strain curves were obtained after finishing a total of 70,000 steps of simulations. Force was calculated by

†Large-scale Atomic/Molecular Massively Parallel Simulator, http://lammps.sandia.gov/

*Visual Molecular Dynamics, http://www.ks.uiuc.edu/Research/vmd/ 
summing up all the forces applied to the top-layer-atoms. The buckling load and the critical strain were depicted as the load and strain of the point at which the curve starts to drop. The Young's modulus was computed from the slope of the linear part.

\section{Result and Discussion}

The dimensions of each CNT after energy minimization are shown in Table 2 and the shapes of nanotubes are shown in Fig. 2.

Table 2. Dimensions of CNTs after energy minimization.

\begin{tabular}{c|ccc}
\hline Chiral vector & $D(\AA)$ & $L(\AA)$ & Aspect ratio $(L / D)$ \\
\hline$(8,8)$ & 11.28 & 82.12 & 7.28 \\
$(14,0)$ & 11.414 & 78.2552 & 6.856 \\
$(6,10)$ & 11.5804 & 80.95 & 6.99 \\
\hline
\end{tabular}

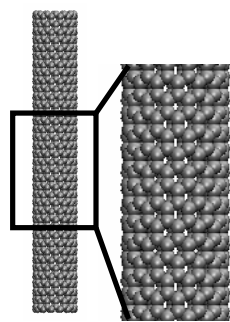

(a)

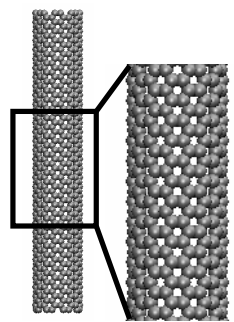

(b)

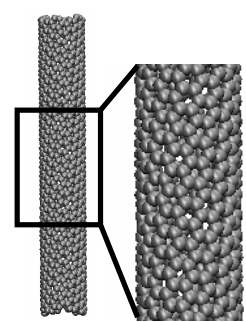

(c)

Fig. 2. Shapes of CNTs after energy minimization; (a) $(8,8)$ armchair CNT, (b) $(14,0)$ zigzag CNT, (c) $(6,10)$ chiral CNT.

The length of each CNT increases after minimization as $\mathrm{C}-\mathrm{C}$ bond length increases from $1.42 \AA$ to $1.45 \AA$. The length of the $\mathrm{C}-\mathrm{C}$ bond in a CNT measured by Wildoer et $a l .{ }^{17}$ is $1.42 \AA$, and the discrepancy between the measurement and simulation results is $2 \%$, which signifies that the Tersoff potential simulates the $\mathrm{C}-\mathrm{C}$ bond in CNTs reliably.

Force-strain curves obtained from the buckling simulation are shown in Fig. 3. Forces increase linearly to the strain of around 0.05 , then drops abruptly, which implies that current simulation describes buckling behaviors properly. The maximum force is the buckling load, and the corresponding strain is the critical strain. The buckling load and the critical strain are represented in Table 3. The Young's modulus was calculated from the slope of the linear part of the curves. The stress was calculated by dividing the force by the section area of the CNTs as in Eq. (3). $D$ is the diameter of CNTs and the layer thickness $t$ was assumed to be $3.4 \mathrm{~nm}^{18}$

$$
A=D t \pi
$$

Table 3. Critical strain, Buckling Load, and Young's modulus of CNTs.

\begin{tabular}{cccc}
\hline Chiral vector & Critical strain $\left(10^{-2}\right)$ & Buckling Load $(n N)$ & $E(T P a)$ \\
\hline$(8,8)$ & 5.34 & 87.1 & 1.38 \\
$(14,0)$ & 5.37 & 89.5 & 1.39 \\
$(6,10)$ & 4.70 & 75.8 & 1.32 \\
\hline
\end{tabular}




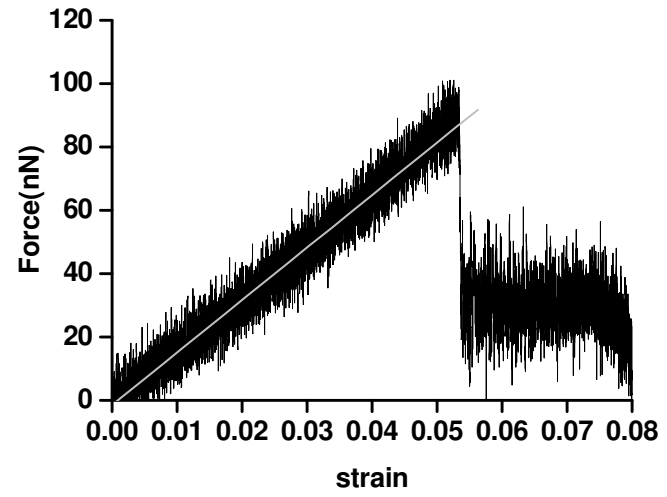

(a)

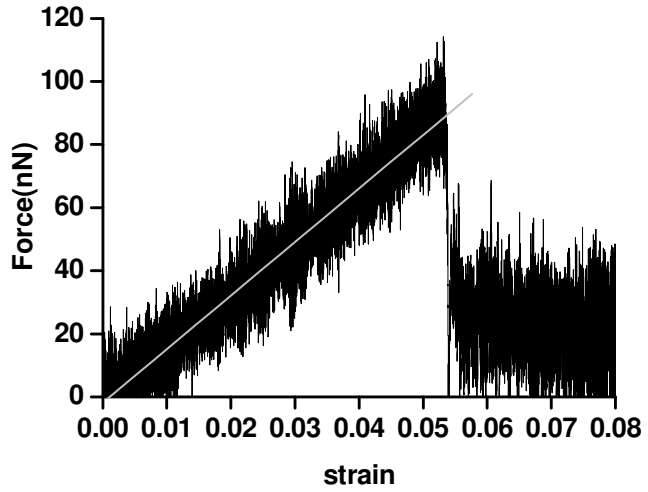

(b)

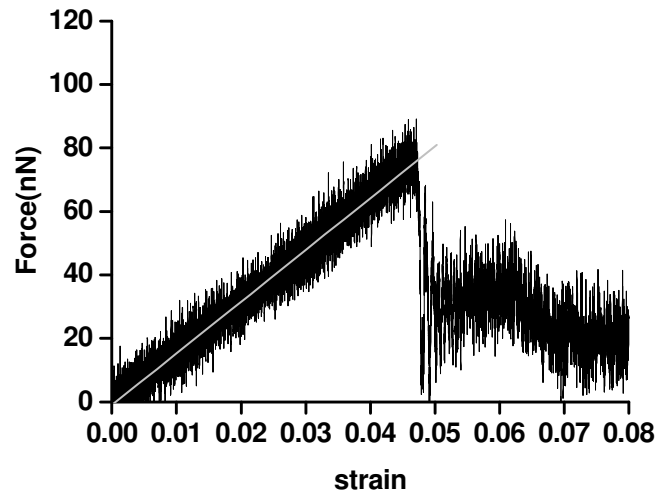

(c)

Fig. 3. Force-strain curve obtained from the buckling simulation, gray lines indicate linear fitting of the force-strain curves; (a) $(8,8)$ armchair CNT, (b) $(14,0)$ zigzag CNT, (c) $(6,10)$ chiral CNT.

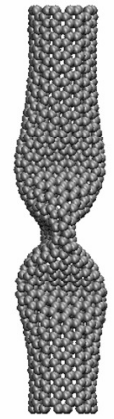

(a)

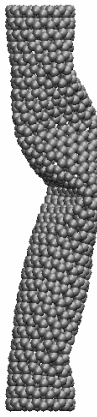

(b)

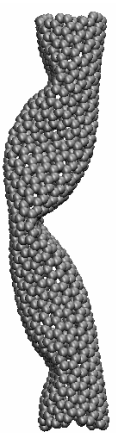

(c)

Fig. 4. Shapes of CNTs under axial loading at the strain of 0.06 ; (a) $(8,8)$ armchair CNT, (b) $(14,0)$ zigzag CNT, (c) $(6,10)$ chiral CNT. 
A zigzag CNT has the highest value of the critical strain, the buckling load and the Young's modulus, and a chiral CNT has the lowest of them, which was shown by $\mathrm{Lu}^{11}$ as well. The differences in the Young's modulus among the nanotubes are approximately $5 \%$. However, the difference in the buckling load among them is around $15 \%$. The effect of helicity on the buckling load is three times larger than that on the Young's modulus. The Young's modulus for nanotubes is around 1.3TPa, which is consistent with the value reported by other researchers. ${ }^{18}$ The shapes of the nanotubes at the strain of 0.06 are shown in Fig. 4. An armchair CNT shows a diamond buckling mode with two lobes, where as a zigzag CNT shows global bending with concentrated local deformation, and a chiral CNT squashed entirely. Different buckling modes can be the reason for the discrepancy in the buckling load of CNTs.

\section{Conclusion}

Molecular dynamics simulations of CNTs under axial compressive load were carried out for SWCNTs with different chiral vector to investigate the effect of the helicity on the buckling behavior of SWCNTs. The buckling load, the critical strain and the Young's modulus were calculated from the force-strain curves. The effect of the helicity of a SWCNT on the buckling load and the critical strain were newly investigated. SWCNTs with around $1.1 \mathrm{~nm}$ thickness and $80 \mathrm{~nm}$ length have the buckling load around $85 \mathrm{nN}$, the critical strain around 0.05 , and the Young's modulus about 1.3TPa. A zigzag nanotube has the largest Young's modulus, critical strain, and buckling load, followed by an armchair, and a chiral nanotube. The differences in the Young's modulus among the SWCNTs with different chiral vectors are slight, but the differences in the buckling load are up to $15 \%$. The shapes of the CNTs at the strain of 0.06 are different from each other. The discrepancy in the buckling load may result from the different buckling mode.

\section{References}

1. S. Iijima, Nature 354, 56 (1991).

2. M. Meyyappan, Carbon Nanotubes: Science and Applications (CRC Press, 2004).

3. M.M.J. Treacy, T.W. Ebbesen, and J.M. Gibson, Nature 381, 678 (1996).

4. B.I. Yakobson, C.J. Brabec, and J. Bernholc, Phys. Rev. Lett. 76, 2511 (1996).

5. N. Hamada, S. Sawada, and A. Oshiyama, Phys. Rev. Lett. 68, 1579 (1992).

6. J.P. Lu, Phys. Rev. Lett. 74, 1123 (1995).

7. T. Ozaki, Y. Iwasa, and T. Mitani, Phys. Rev. Lett. 84, 1712 (2000).

8. M.J. Buehler, Y. Kong, and H. Gao, J. Eng. Mater. Technol. 126, 245 (2004).

9. G. Dereli and C. Özdoğan, Phys. Rev. B 67, 035416 (2003).

10. T.W. Odom et al., Nature 391, 63 (1998).

11. J.P. Lu, Phys. Rev. Lett. 79, 1297 (1997).

12. J. Tersoff, Phys. Rev. B 37, 6991 (1988).

13. J.M. Haile, Molecular dynamics simulation: elementary methods (Wiley, 1992).

14. Z. Yao et al., Computational Materials Science 22, 180-184 (2001).

15. M.S. Daw and M.I. Baskes, Phys. Rev. B 29, 6443 (1984).

16. M.W. Finnis and J.E. Sinclair, Philosophical Magazine A 50, 45 (1984).

17. J.W.G. Wildoer et al., Nature 391, 59 (1998).

18. S. Akita and M.N.A.Y. Nakayama, Jpn. J. Appl. Phys. 45, 5586-5589 (2006). 